\title{
Replik: Kultur- und Bildungskritik und die Suche nach einer neuen Lebensform
}

\section{Michael Hampe}

\section{(2) OpenEdition \\ 1 Journals}

\section{Electronic version}

URL: http://journals.openedition.org/ejpap/2181

DOI: 10.4000/ejpap.2181

ISSN: 2036-4091

\section{Publisher}

Associazione Pragma

\section{Electronic reference}

Michael Hampe, «Replik: Kultur- und Bildungskritik und die Suche nach einer neuen Lebensform », European Journal of Pragmatism and American Philosophy [Online], XII-2 | 2020, Online since 14

December 2020, connection on 15 December 2020. URL : http://journals.openedition.org/ejpap/2181 ; DOI : https://doi.org/10.4000/ejpap.2181

This text was automatically generated on 15 décembre 2020 .

\section{$\Theta \Theta \Theta$}

Author retains copyright and grants the European Journal of Pragmatism and American Philosophy right of first publication with the work simultaneously licensed under a Creative Commons AttributionNonCommercial-NoDerivatives 4.0 International License. 


\title{
Replik: Kultur- und Bildungskritik und die Suche nach einer neuen Lebensform
}

\author{
Michael Hampe
}

1 Yvonne Hütter-Almerigi und Dirk Jörke haben wichtige Einwände zu meinem Essay über eine mögliche Dritte Aufklärung vorgetragen. Ich danke ihnen sehr für die Aufmerksamkeit, die sie meinem Text haben zukommen lassen und für die Mühe, die sie sich mit der Entwicklung ihrer wertvollen Kritik gemacht haben.

2 Ich weiss nicht, ob ich auf ihre Kritiken angemessen regieren kann, weil mir Kompetenzen in den Bereichen der empirischen Sozialforschung und der Ökonomie fehlen, die für die Beantwortung mancher ihrer Fragen wohl nötig wären. Diese, scheint mir, müsste ich beispielsweise haben, um auf Einwände zu reagieren, die beide im Zusammenhang mit der Frage entwickeln, welche Rolle die Herstellung von Verteilungsgerechtigkeit in unserer Gesellschaft für die Möglichkeit der Realisierung einer Dritten Aufklärung zu spielen hätte. Auch bei der Frage von Jörke, was wirklich die Ursache des Aufstiegs der populistischen Parteien ist: grossangelegte Täuschungen oder Unzufriedenheiten mit der eigenen wirtschaftlichen Lage (bspw. in Virgina, wo das Trumpsche Wahlergebnis von 2016 fulminant war, worauf Jörke verweist), kann ich keine empirisch begründete Antwort geben, weil ich es schlicht nicht weiss, keine Daten der empirischen Sozialforschung zur Verfügung habe.

Das ist wohl ein Grundproblem mit einem philosophischen Essay, das er nicht unbedingt auf empirischer Sozialforschung beruht, sondern häufig hypothetischen Charakter hat. Ich würde jedoch auch nach Jörkes Kritik weiter die Hypothese ventilieren wollen, dass beides eine Rolle spielte: eine mangelnde Orientierung an etablierten Wahrheitspraktiken, sowohl bei den Trumpwählerinnen und -wählern und bei den Menschen, die den Brexit befürwortet haben, wie auch ein Zorn darüber, dass sich niemand um die eigenen wirtschaftlichen Nöte kümmert, ein Zorn, der, wie es Michael Moore formuliert hat, dazu führen mag, dass man einen "Molotow Cocktail" mit der Aufschrift "Trump" bereit ist, ins "Weiße Haus" zu schleudern, um "das 
System," das einen im Stich gelassen hat, anzuzünden. Es geht hier ja nicht um ein "Entweder-Oder": Entweder eine grosse Zahl der Wählerschaft wird belogen oder sie wird wirtschaftlich im Regen stehen gelassen. Beides ist ja der Fall; Menschen werden systematisch wirtschaftlich benachteiligt und sie werden belogen, sowohl darüber, warum sie benachteiligt sind, wie auch darüber, welche Medien Wahrheitspraktiken realisieren, die es erlauben, sich verlässliche Informationen zu besorgen. Den Begriff der Wahrheitspraktik habe ich in anderen Zusammenhängen konkretisiert. Er hat nichts mit Platonischen Vorstellungen einer ewigen Wahrheit zu tun, im Gegenteil. ${ }^{1}$ Es wird in "unseren" Gesellschaften gegenwärtig sowohl eine immer weitergehende Verteilungsungerechtigkeit realisiert wie auch eine systematische Demontage des Vertrauens in bewährte Wahrheitspraktiken, die durch nichts Tauglicheres ersetzt werden. Letztlich muss ich hoffen, dass die empirische Sozialforschung herausbringen wird, welche Motivlage sich aus diesen Prozessen bei der Mehrheit der betreffenden Bevölkerung entwickelt hat.

Sowohl in Yvonne Hütter-Almerigis wie in Dirk Jörkes Kritik nehme ich ein Unbehagen an meiner Verwendung des Begriffs einer aufgeklärten Kultur wahr. Beide wollen mich, wenn ich recht verstehe, darauf hinweisen, dass ich mich mit meinen Anliegen doch mehr um die "harten" politischen und ökonomischen Fakten und weniger um allgemeine kulturelle Rahmenbedingungen kümmern sollte. Allerdings habe ich tatsächlich den Eindruck, dass die politischen und ökonomischen Probleme der meisten "westlichen" Gesellschaften gegenwärtig auf solche der Kultur, ja vielleicht sollte ich sogar sagen: der Lebensform zurückzuführen sind.

5 Entsprechend scheint es mir nicht angemessen, die Chancengleichheit und Verteilungsgerechtigkeit in dem bestehenden Bildungs-, Arbeits- und Wirtschaftssystem zu verbessern. Ebenso wenig erscheint es mir sinnvoll, einen nachhaltigeren Umgang mit den natürlichen Ressourcen anzustreben, damit es Menschen möglich ist, diese weiterhin auszubeuten. Eine Verbesserung der Chancengleichheit, der Verteilungsgerechtigkeit und ein nachhaltigeres Wirtschaften würden m.E. die Kultur oder die Lebensform, in der die Menschen gegenwärtig leben, nicht wirklich ändern, es wären Anpassungen innerhalb der bestehenden Kultur oder Lebensform, die eine ist, die in bestimmten Hinsichten zur Selbstkorrektur unfähig geworden ist. Von einer Dritten Aufklärung würde ich mir daher eine grundlegende Änderung der gegenwärtigen Kultur- und Lebensform erwarten.

6 So glaube ich nicht, dass eine Deweysche Absage an das Streben nach absoluter Gewissheit in unserer gegenwärtigen epistemischen Kultur vollzogen worden ist. Die Metapher von Plastiktüten und Rindsledertaschen für Meinungen und Wissen ist von mir nicht als eine Restituierung der alten Unterscheidung von doxa und episteme oder als Versuch gedacht, eine "unveränderliche Sphäre des Wissens" wiedereinzuführen, wie Jörke meint. Denn auch Rindsledertaschen gehen kaputt, halten vielleicht ein Leben, aber nicht ewig. Dass es das Charakteristikum aufgeklärten Wissens ist, selbstkritisch und damit revidierbar zu sein und dass es sich in eine Vielfalt von Wahrheitspraktiken ausdifferenziert hat, die durch keine letzte philosophische Wahrheit kontrolliert werden, ist erstens kein Platonismus und zweitens weder im öffentlichen Bewusstsein, noch in unserem Bildungssystemen angekommen. Diese sind vielmehr immer noch von der Dichtomie: entweder absolute Gewissheit des Wissens oder Relativismus geprägt und nicht von einer Einübung in eine Vielfalt von revidierbaren Wahrheitspraktiken. 
7 Dewey hat m.E. Recht gehabt als er glaubte, dass sich eine Gesellschaft vor allem über ihr Bildungssystem verändern muss. Nur wenn andere Menschen in anderen Schulen entstehen, kann auch die Gesellschaft eine andere werden. Einfach die Institutionen zu verändern, reicht nicht, wenn sie von Menschen mit den "alten" Gewohnheiten und Erwartungen "betrieben" werden. Gegenwärtig dienen Schulen aber dazu, Kinder dafür "fit" zu machen in dieser Gesellschaft erfolgreich zu sein und nicht eine neue aufzubauen. Eltern hätten Angst ihre Kinder auf Schulen zu schicken, die sie nicht auf diese Gesellschaft vorbereiten, ihnen nicht möglichst hohe Berufschancen in dieser Gesellschaft verschaffen. Ich habe dagegen auf Seite 83 meines Essays gefordert: "Primäres Ziel von Bildung ist nicht die Steigerung von Karrierechancen, die Suche nach Gewissheit, das Speichern von Informationen oder eine abstrakte Entscheidungskompetenz, sondern der Erwerb von Kreativität, die das individuelle Leben zu einem sinnvollen machen und Gemeinschaften eine kulturelle Gestalt $\mathrm{zu}$ geben vermag..." Nur kreative selbstbewusste Menschen können aus einer Kritik der gegenwärtigen Lebensform die gesellschaftlichen Konsequenzen ziehen, die eventuell $\mathrm{zu}$ einer neuen Lebensform in the long run führen.

8 So, wie es in einer aufgeklärten Kultur oder Lebensform meines Erachtens eines allgemeinen Bewusstseins der Unmöglichkeit absoluter Gewissheit bedürfte, ebenso bedürfte es einer Ausdifferenzierung von Bildungs- oder Weisheitspraktiken, der Praktiken der sinnvollen Lebensführung, die ohne absolute Gewissheiten auskommen. Die existenziellen und bildungsinstitutionellen Konsequenzen einer solchen Absage an absolute Gewissheiten sind meines Erachtens jedoch noch gar nicht durchdacht. Was heisst es, seinem persönlichen und dem gemeinschaftlichen Leben eine Orientierung zu geben, die Bedürfnisse nach intensiver Erfahrung zu befriedigen, wenn es kein letztes epistemisches Fundament für diese Orientierung gibt, weder in der Wissenschaft noch in einer Religion? Was heisst es, jemanden zu bilden, wenn alle Wissensbestände potentiell revidierbar sind und auf keinem Gewissheitsfundament ruhen? Wie schafft man Vertrauen in bewährte Wahrheitspraktiken, ohne auf absolute Gewissheiten Bezug zu nehmen?

9 Weder in Deweys Erziehungsphilosophie noch in der Diskursethik gibt es, soweit ich beide überblicke, Antworten auf diese Fragen. Die zweite Aufklärung hatte, mit Raymond Geuss gesprochen, ein unaufgeklärtes Verhältnis zu ihrem eigenen Vernunftund Wahrheitsverständnis, weil sie glaubte, dass sich "die Vernunft" und "die Wahrheit" schon durchsetzen werden. ${ }^{2}$ Die Einsicht, dass es "die Vernunft" nicht gibt, sondern nur eine Reihe von mehr oder weniger gut begründeten Praktiken des Zusammenlebens und der Erkenntnis, die auch kein "System" bilden, dass es "das Gute" und "die Wahrheit" nicht gibt, sondern nur eine Reihe von mehr oder weniger gut begründeten Lebens- und Wahrheitspraktiken hat $\mathrm{zu}$ der falschen Überzeugung geführt, man könne auf Begründungen und Wahrheiten überhaupt verzichten und Beliebiges veranstalten oder glauben. Dieser falsche Glaube ist meines Erachtens Symptom für die Krise einer Kultur oder Lebensform, die immer noch auf die Fetische der absoluten Gewissheit und des notwendigen "Fortschritts" fokussiert ist, die also die eigenen Aufklärungsideale noch nicht umsetzen konnte. Diese Fetische aufzugeben, würde in meinen Augen bedeuten, einen kulturellen Wandel, einen Wandel in der Lebensform zu vollziehen, der, anders als Jörke meint, gerade nicht zum Platonismus zurückführen, sondern eher einen existentiellen und pragmatischen Pyrrhonismus wiederbeleben würde (wie er etwa in den Arbeiten von Michael Williams angedeutet 
wird $\left.{ }^{3}\right)$, den es gesellschaftlich m.E. bisher nie gegeben hat und dessen politische und ökonomische Konsequenzen mir noch unklar sind.

\section{NOTES}

1. Michael Hampe, (2019), "Wahrheitspraktiken," in Heiner Hastedt (hrg.), Deutungsmacht von Zeitdiagnosen. Interdisziplinäre Perspektiven, Bielefeld, transcript Verlag, 49-66.

2. Vgl. Raymond Geuss, (2020), "Enlightenment, Genealogy and the Historicality of Concepts," Kapitel 3 von: Who needs a World-View?, Cambridge/Ma., Harvard University Press.

3. Michael Williams, (1988), "Scepticism Without Theory," Review of Metaphysics 41, 547-88.

\section{AUTHOR}

MICHAEL HAMPE

ETH Zürich

hampe[at]phil.gess.ethz.ch 